\title{
Extensions of Spaces With Cylindrical Measures and Supports of Measures Determined by the Lévy Laplacian
}

\author{
L. Accardi and O. G. Smolyanov
}

UDC 517.518

\begin{abstract}
Extensions of noncountably additive (cylindrical) measures are described, and examples of Hilbert supports of the Lévy-Gauss measure are given.

KEY WORDS: Lévy Laplacian, cylindrical measure, extension of a measurable space, extension of a space with measure, Lévy-Gauss measure, Gaussian measure, Lévy trace, Volterra-Gross Laplacian.
\end{abstract}

Recently, the operator known as the Lévy Laplacian has become popular thanks to the discovery [1, 2] of the equivalence between the Yang-Mills equations and the equation with this Laplacian. The authors [3-6] described a new approach to the study of differential equations with the Lévy Laplacian; the approach was based on the application of the Lévy-Gauss measure, which was introduced in these works as a noncountably additive cylindrical Gaussian measure defined on some nonseparable Hilbert space and admitting no countably additive Hilbert extensions (because the space was nonseparable); all definitions will be given later. The question on the existence of a Hilbert support for the Lévy-Gauss measure remained open.

In this paper, extensions of noncountably additive (cylindrical) measures are systematically described and examples of Hilbert supports for the Lévy-Gauss measure are given.

\section{§1. Preliminaries}

A measurable space is a pair $(\Omega, \mathfrak{A})$, where $\Omega$ is a set and $\mathfrak{A}$ is an algebra of its subsets (this definition differs from the standard one). A measure on $(\Omega, \mathfrak{A})$ (or, shorter, on $\Omega$, or on $\mathfrak{A}$ ) is a finitely additive complex-valued bounded function on $\mathfrak{A}$.

Let $\left(\Omega_{j}, \mathfrak{A}_{j}\right)$ with $j=1,2$ be measurable spaces.

Definition 1. The space $\left(\Omega_{2}, \mathfrak{A}_{2}\right)$ is called a (measurable) extension of the space $\left(\Omega_{1}, \mathfrak{A}_{1}\right)$ if

(a) $\Omega_{1} \subset \Omega_{2}$

(b) $\mathfrak{A}_{1}=\left\{A \cap \Omega_{1}: A \in \mathfrak{A}_{2}\right\}$;

(c) $\left[A \cap \Omega_{1}=B \cap \Omega_{1}\right] \Longrightarrow A=B$ for all $A, B \in \mathfrak{A}$.

Remark 1. If $\left(\Omega_{1}, \mathfrak{A}_{1}\right)$ is isomorphic to a measurable space $\left(\Omega_{3}, \mathfrak{A}_{3}\right)$, then any extension of the space $\left(\Omega_{3}, \mathfrak{A}_{3}\right)$ is also called an extension of $\left(\Omega_{1}, \mathfrak{A}_{1}\right)$.

Remark 2. Conditions (b) and (c) determine an isomorphism of the algebras $\mathfrak{A}_{1}$ and $\mathfrak{A}_{2}$; we call it canonical.

Definition 2. Measurable spaces $\left(\Omega_{j}, \mathfrak{A}_{j}\right)$ with $j=1,2$ are called versions of each other if there exists a measurable space that is an extension of each of them.

Note that, by Remark 2 , the algebras $\mathfrak{A}_{1}$ and $\mathfrak{A}_{2}$ are then (canonically) isomorphic.

Throughout the following, the field of scalars is assumed to be the field of real numbers.

Translated from Matematicheskie Zametki, Vol. 64, No. 4, pp. 483-492, October, 1998.

Original article submitted May 13, 1997. 
Example 1. Consider some vector snace $E$ and the vector space $G$ of linear functionals on $E$. For each subset $G_{0} \in G$, we define $\mathfrak{A}\left(E, G_{0}\right)$ as the algebra of all $G_{0}$-cylindrical subsets of $E$; thus $A \in \mathfrak{A}\left(E, G_{0}\right)$ if and only if there exist a positive integer $n$, elements $g_{1}, \ldots, g_{n} \in G_{0}$, and a Borel subset $B$ of the space $\mathbb{R}^{n}$ such that $A=\left\{x \in E:\left(g_{1}(x), \ldots, g_{n}(x)\right) \in B\right\}$. For each subset $E_{0}$ of $E, \mathfrak{A}\left(G, E_{0}\right)$ is the algebra of all $E_{0}$-cylindrical subsets $G$; it is defined similarly.

Consider a vector space $E$. Let $G_{j}$, where $j=1,2$, be vector spaces consisting of linear functionals on $E$ and being in natural duality with $E$ (this means that for any nonzero $x \in E_{0}$ there exist $g_{j} \in G_{j}$ such that $g_{j}(x) \neq 0$ for $\left.j=1,2\right)$. Suppose that $G$ is some vector space of linear functionals on $E$ that contains the set $G_{1} \cup G_{2}$. Then the measurable space $(G, \mathfrak{A}(G, E))$ is an extension of each of the spaces $\left(G_{j}, \mathfrak{A}\left(G_{j}, E\right)\right)$; therefore, the spaces $\left(G_{j}, \mathfrak{A}\left(G_{j}, E\right)\right), j=1,2$, are versions of each other.

We say that a measure space is a set $(\Omega, \mathfrak{A}, \nu)$, where $(\Omega, \mathfrak{A})$ is a measurable space and $\nu$ is a measure on $\mathfrak{A}$ (this definition also differs from the standard one).

Definition 3. Suppose that $\left(\Omega_{j}, \mathfrak{A}_{j}, \nu_{j}\right)$, where $j=1,2$, are measure spaces. The space $\left(\Omega_{2}, \mathfrak{A}_{2}, \nu_{2}\right)$ is called an extension of $\left(\Omega_{1}, \mathfrak{A}_{1}, \nu_{1}\right)$ if $\left(\Omega_{2}, \mathfrak{A}_{2}\right)$ is an extension of the measurable space $(\Omega, \mathfrak{A})$ and $\nu_{2} A=\nu_{1}\left(A \cap \Omega_{1}\right)$ for each $A \in \mathfrak{A}_{2}$ (this equality justifies the term extension of a measure space). If $\left(\Omega_{2}, \mathfrak{A}_{2}, \nu_{2}\right)$ is an extension of $\left(\Omega_{1}, \mathfrak{A}_{1}, \nu_{1}\right)$, then $\nu_{2}$ is called an extension of $\nu_{1}$.

Note that in Definition $3 \nu_{2}$ is the image of $\nu_{1}$ under the canonical embedding $F: \Omega_{1} \rightarrow \Omega_{2}$.

Definition 4. Measure spaces $\left(\Omega_{j}, \mathfrak{A}_{j}, \nu_{j}\right), j=1,2$, are called versions of each other if they have a common extension; the measures $\nu_{1}$ and $\nu_{2}$ are then also called versions of each other. If, in addition, $\nu_{2}$ is countably additive, then $\Omega_{2}$ is called a support of $\nu_{1}$.

Note that it may happen that $\Omega_{2}$ is a support of $\nu_{1}$, but nevertheless $\Omega_{2} \cap \Omega_{1}=\varnothing$.

Remark 3. If measurable spaces $\left(\Omega_{j}, \Omega_{j}\right)$ with $j=1,2$ are versions of each other, then the algebras $\mathfrak{A}_{1}$ and $\mathfrak{A}_{2}$ are canonically isomorphic, and therefore, for any measure $\nu_{1}$ on $\mathfrak{A}_{1}$, there exists a (unique) measure $\nu_{2}$ on $\mathfrak{A}_{2}$ such that the spaces $\left(\Omega_{1}, \mathfrak{A}_{1}, \nu_{1}\right)$ and $\left(\Omega_{2}, \mathfrak{A}_{2}, \nu_{2}\right)$ are versions of each other ( $\nu_{2}$ is the (canonical) image of $\nu_{1}$ under the corresponding isomorphism). Thus $\Omega_{2}$ is a support of the measure $\nu_{1}$ if the canonical image of this measure is a countably additive measure on $\mathfrak{A}_{2}$.

\section{§2. Countably additive extensions of measures}

Theorem 1 (cf. [7]). Any measurable space $(\Omega, \mathfrak{A})$ has a (measurable) extension $\left(\Omega_{1}, \mathfrak{A}_{1}\right)$ such that $\Omega_{1}$ is a support of every measure on $(\Omega, \mathfrak{A})$.

Proof. Let $\mathcal{F}$ be the set of all functions that are defined on the set of all subsets of $\Omega$ and range in $\{0,1\}$, and let $\Omega_{1}$ be the set of all functions that are defined on $\mathcal{F}$ and take values in $\{0,1\}$. We denote the set of characteristic functions of all sets from $\mathfrak{A}$ by $\mathcal{F}_{0}$ (thus $\mathcal{F}_{0} \subset \mathcal{F}$ ) and the algebra of all subsets of $\Omega_{1}$ that is generated by sets of the form $\left\{\alpha \in \Omega_{1}: \alpha(f)<1\right\}$ with $f \in \mathcal{F}_{0}$ by $\mathfrak{A}_{1}$. The map $\Omega \ni x \mapsto[F \ni f \mapsto f(\{x\})] \in \Omega_{1}$ is an embedding of $\Omega$ into $\Omega_{1}$. Let $\left(\Omega^{\prime}, \mathfrak{A}^{\prime}\right)$ be the image of $(\Omega, \mathfrak{A})$ under this embedding. Then $\left(\Omega_{1}, \mathfrak{A}_{1}\right)$ is an extension of the space $\left(\Omega^{\prime}, \mathfrak{A}^{\prime}\right)$ and therefore, of the space $(\Omega, \mathfrak{A})$. If $\nu$ is a measure on $\mathfrak{A}$, then its image in $\left(\Omega_{1}, \mathfrak{A}_{1}\right)$ is countably additive by the Daniel-Kolmogorov theorem.

Next, for a vector space $E$, we write $E^{*}\left(E^{\prime}\right)$ to denote the algebraically (topologically) dual space, i.e., the space of all linear (linear continuous) functionals on $E$. If $E$ is a vector space, $G$ is a vector space of some linear functionals on $E, G_{0} \subset G$, and $E_{0} \subset E$, then a $G_{0}$-cylindrical measure on $E$ is an arbitrary measure on $\mathfrak{A}\left(E, G_{0}\right)$ whose restriction to any algebra $\mathfrak{A}(E, T)$ of sets with finite $T \subset G_{0}$ is countably additive; similarly, an $E_{0}$-cylindrical measure on $G$ is an arbitrary measure on $\mathfrak{A}\left(G, E_{0}\right)$ whose restriction to any algebra $\mathfrak{A}(G, T)$ of sets with finite $T \subset E_{0}$ is countably additive. If $E$ is a vector space, $E_{0} \subset E$, spaces $G_{j} \subset E^{*}$ with $j=1,2$ separate points in $E$ (i.e., are in canonical duality with $E$ ), and $\nu$ is an $E_{0}$-cylindrical measure on $G_{1}$, then the canonical image of this measure in $\mathfrak{A}\left(G_{2}, E_{0}\right)$ is an $E_{0}$-cylindrical measure on $G_{2}$ (and is a version of the measure $\nu$ ). 
Theorem 2. Suppose that $E$ is a vector space and $G$ is a vector space of some linear functionals on $E$ that separate points in $E$. Let $E_{0}$ be a linearly independent subset of $E$ such that $g_{1}=g_{2}$ whenever $g_{1}, g_{2} \in G$ and $g_{1}(x)=g_{2}(x)$ for all $x \in E_{0}$. Then the vector space $G_{0}$ of all real-valued functions $f$ on $E_{0}$ for which the sets $\left\{x \in E_{0}: f(x) \neq 0\right\}$ are at most countable is a support of any $E_{0}$-cylindrical measure on $G$.

Proof. Let $G_{1}$ be the vector space of all (real-valued) functions on $E_{0}$. Then $E_{0}$ can be considered a subset of each of the spaces $G_{1}^{*}$ and $G_{0}^{*}$, which allows us to define the algebras $\mathfrak{A}\left(G_{1}, E_{0}\right)$ and $\mathfrak{A}\left(G_{0}, E_{0}\right)$ of sets. Since $\left(G_{1}, \mathfrak{A}\left(G_{1}, E_{0}\right)\right)$ is an extension of both measurable spaces $\left(G_{0}, \mathfrak{A}\left(G_{0}, E_{0}\right)\right)$ and $\left(G, \mathfrak{A}\left(G, E_{0}\right)\right)$, it follows that these two spaces are versions of each other. If $\nu_{0}$ is the canonical image in $\mathfrak{A}\left(G_{0}, E_{0}\right)$ of an $E_{0}$-cylindrical measure $\nu$ on $G$, then $\nu_{0}$ is countably additive by the Daniel-Kolmogorov theorem. This completes the proof of Theorem 2 .

Note that $G_{1}$ is also a support of any $E_{0}$-cylindrical measure on $G$, because $G_{1} \supset G_{0}$.

Recall that if $E$ and $G$ are vector spaces, $G \subset E^{*}, E_{0} \subset E$, and $\nu$ is an $E_{0}$-cylindrical measure on $G$, then the Fourier transform of $\nu$ is the function $F \nu$ on $E_{0}$ defined by

$$
F \nu(x)=\int e^{\mathrm{i} g(x)} \nu(d g)
$$

It is known [7] that if $E_{0}$ is a vector subspace of $E$, then the function $F \nu$ uniquely determines the measure $\nu$.

Recall also that if $E_{0}$ is a vector subspace of $E$, then an $E_{0}$-cylindrical measure $\nu$ is called Gaussian (with zero mean) if

$$
F \nu(x)=\exp \left(-\frac{1}{2} b(x)\right)
$$

where $b$ is a (nonnegative definite) quadratic form on $E_{0}$ (the correlation functional associated with the measure $\nu$ ), i.e., $b(x)=(B x)(x)$, where $B$ is a linear map of $E_{0}$ into $G$ called the correlation operator (or covariance) of the measure $\nu$.

Remark 4. Suppose that $G_{j}$ and $E$ are vector spaces, $G_{j} \subset E^{*}$, each $G_{j}$ separates points in $E$, and $\nu_{j}$ are $E$-cylindrical measures on $G_{j}(j=1,2)$. These measures are versions of each other if and only if $F \nu_{1}=F \nu_{2}$.

Thus the Fourier transform of an $E$-cylindrical measure $\nu$ uniquely determines all $E$-cylindrical measures that are versions of $\nu$.

Theorem 3 (cf. [8]). Suppose that $H_{j}, j=1,2$, are Hilbert spaces, $H_{1}$ is a dense vector subspace of $H_{2}$, and the canonical embedding $p: H_{1} \rightarrow H_{2}$ is Hilbert-Schmidt. Let $\nu_{1}$ be an $H_{1}^{\prime}$-cylindrical measure on $H_{1}$ with a continuous Fourier transform. Then $H_{2}$ is a support of the restriction of $\nu_{1}$ to the algebra of $p^{*} H_{2}^{\prime}$-cylindrical subsets of $H_{1}$ (which is a subalgebra of $\mathfrak{A}\left(H_{1}, H_{1}^{\prime}\right)$ ).

Proof. It suffices to show that the $H_{2}^{\prime}$-cylindrical measure on $H_{2}$ that is the image of $\nu_{1}$ under $p$ is countably additive. But $F\left(\nu p^{-1}\right)(x)=F \nu\left(p^{*} x\right)$ and since $F \nu$ is continuous, it follows that the function $F\left(\nu p^{-1}\right)$ is continuous with respect to the Sazonov topology.

Remark 5. For a Gaussian measure $\nu$, Theorem 3 can be strengthened. Namely, $\mathrm{H}_{2}$ is then a support not only of the restriction of the measure $\nu$ but also of the measure itself. Indeed, if $\nu$ is Gaussian, then every functional $f \in H_{1}^{\prime}$ can be (uniquely) extended to a linear measurable functional on $\left(H_{2}, \sigma_{\nu}\right)$; here $\sigma_{\nu}$ is the $\sigma$-algebra where the Lebesgue extension of the measure $\nu_{2}=\nu p^{-1}$ is defined. Moreover, each linear measurable functional on $\left(H_{2}, \sigma_{\nu}\right)$ can be obtained in this manner. Next, let $H_{2}^{m}$ be the set of representatives of all equivalence classes of measurable linear functionals on $\left(H_{2}, \sigma_{\nu}\right)$. Taking into account the preceding, we can assume that $H_{2}^{m}=H_{1}^{\prime}$; this means that $\left(H_{2}, \mathfrak{A}\left(H_{2}, H_{2}^{m}\right)\right)$ is an extension of $\left(H_{1}, \mathfrak{A}\left(H_{1}, H_{1}^{\prime}\right)\right)$. The canonical image of the measure $\nu_{1}$ in $\mathfrak{A}\left(H_{2}, H_{2}^{m}\right)$ is countably additive (it coincides with the restriction of the Lebesgue extension of $\nu_{2}$ to $\mathfrak{A}\left(H_{2}, H_{2}^{m}\right)$ ), which implies that $H_{2}$ is a support of the measure $\nu_{1}$.

The following example can be viewed as standard. 
Example 2. Consider the space $H_{1}$ of all absolutely continuous functions $\varphi$ on $[0,1]$ that vanish at zero and have square integrable derivatives; the norm on $H_{1}$ is defined by

$$
\|\varphi\|^{2}=\int_{0}^{1}\left|\varphi^{\prime}(t)\right|^{2} d t
$$

(i.e., $\left.H_{1}=W_{2}^{1}[0,1]\right)$. Let $\nu$ be the canonical Gaussian measure on $H_{1}$, i.e., the measure such that

$$
F \nu(\varphi)=\exp \left(-\frac{1}{2}\|\varphi\|^{2}\right)
$$

Then the standard Wiener measure on $C[0,1]$ is a (countably additive) extension of $\nu$, as follows from Remark 5, because the space of measurable linear functionals on $C[0,1]$ equipped with the Wiener measure is isomorphic to the space of measurable linear functionals on $H_{2}=\mathcal{L}_{2}(0,1)$ equipped with the extension of $\nu$ described in Remark 5.

According to Theorem 3, a cylindrical measure can have a "Hilbert support." Below, we define this notion and state a related (negative) result.

Suppose that $E$ is a normed space, $G \subset E^{*}$ is a vector space (separating points in $E$ ), and $\nu$ is an $E$ cylindrical measure on $G$ with continuous Fourier transform (defined on $E$ ). An ( $E$-) Hilbert version of the measure $\nu$ is a $Q$-cylindrical measure $\nu_{Q}$ on some Hilbert space $Q$ (which is identified with its strong dual) such that $\nu_{Q}$ has a continuous Fourier transform and if $\nu_{1}$ is a version of $\nu$ defined on the strong dual $E_{\beta}^{\prime}$ of $E$, then there exists a continuous map $P: E_{\beta}^{\prime} \rightarrow Q$ with dense range such that the measure $\nu_{Q}$ coincides with the image of $\nu$ under $P$.

If $G$ is a vector space and $\nu$ is a $G$-cylindrical Gaussian measure with a nondegenerate correlation functional, then an arbitrary $\left(G,\|\cdot\|_{b}\right)$-Hilbert version of $\nu_{Q}$, where $\|\cdot\|_{b}$ is the norm on $G$ generated by $b$, is called a Hilbert version of $\nu$; if the measure $\nu_{Q}$ is countably additive, then $Q$ is called a Hilbert support of $\nu$.

Proposition 1. If $G$ is a vector space, $\nu$ is a $G$-cylindric Gaussian measure with a (nondegenerate) correlation functional $b$, and the pre-Hilbert space $\left(G,\|\cdot\|_{b}\right)$ is nonseparable, then $\nu$ has no Hilbert support.

This follows from the criterion for countable additivity of Gaussian measures on Hilbert spaces.

\section{§3. Lévy-Gauss measures}

Suppose that $E$ is a (separable) locally convex space, $\|\cdot\|$ is a continuous Hilbert norm on $E$, and $H$ is the completion of the space $(E,\|\cdot\|)$; thus $E \subset H=H^{\prime} \subset E^{\prime}$ is a rigged Hilbert space. Next, let $b=\left(e_{n}\right)$ be an orthonormal basis in $H$ such that it consists of elements of $E$ and for any bounded sequence $\left(c_{n}\right)$ of corimplex numbers the series $\sum c_{n} e_{n}$ converges in $\mathbb{C} \otimes E_{\beta}^{\prime}$. For every dense subset $\Lambda$ of the reals, by $S_{b}^{c \Lambda}$ we denote the linear hull in $\mathbb{C} \otimes E_{\beta}^{\prime}$ of $\left\{\sum e^{i \lambda n} e_{n}\right\}_{\lambda \in \Lambda}$ equipped with the inner product $(\cdot)_{\mathrm{PL}}$ defined by

$$
\left(\sum_{n=1}^{\infty} c_{n} e_{n}, \sum_{n=1}^{\infty} d_{n} e_{n}\right)_{\mathrm{PL}}=\lim _{n \rightarrow \infty} \frac{1}{n} \sum_{j=1}^{n} e_{j} \bar{d}_{j}
$$

in what follows, we call it the Lévy scalar product. The existence of the limits of $c_{n}=e^{i \lambda_{1} n}$ and $d_{n}=e^{i \lambda_{2} n}$ is proved as follows:

$$
\begin{aligned}
\lim _{n \rightarrow \infty} \frac{1}{n} \sum_{j=1}^{n} c_{j} \bar{d}_{j} & =\lim _{n \rightarrow \infty} \frac{1}{n} \sum_{j=1}^{n} e^{i\left(\lambda_{1}-\lambda_{2}\right) j} \\
& =\lim _{n \rightarrow \infty} \frac{1}{n}\left(1-e^{i n\left(\lambda_{1}-\lambda_{2}\right)}\right)\left(1-e^{i\left(\lambda_{1}-\lambda_{2}\right)}\right)^{-1}=0, \quad \lambda_{1} \neq \lambda_{2} .
\end{aligned}
$$


Let $S_{b}^{\Lambda}$ denote the vector subspace of $S_{b}^{c \Lambda}$ consisting of all elements of $S_{b}^{c \Lambda}$ having the form $\sum \alpha_{j} e_{j}$, where $\lambda_{j} \in \mathbb{R}^{1}$ (thus $S_{b}^{c \Lambda}=\mathbb{C} \otimes S_{b}^{\Lambda}$ ); we assume that $S_{b}^{\Lambda}$ is equipped with the inner product induced by $(\cdot)_{\mathrm{PL}}$ (we denote it by the same symbol).

Consider $S_{b}=\bigcup_{\Lambda} S_{b}^{\Lambda}$, where the union is over all countable dense subsets of $\mathbb{R}^{1}$. The inner product $(\cdot)_{\mathrm{PL}}$ determines natural dualities between $S_{b}^{\Lambda}$ and $S_{b}^{\Lambda}$ and between $S_{b}$ and $S_{b}$. In addition, each of these spaces is dual to $E$; the duality is induced by the duality (unrelated to those just mentioned) between $E$ and $E^{\prime}$, because $E^{\prime} \supset S_{b}$. Thus we can assume that $E \subset S_{b}^{*}, E \subset\left(S_{b}^{\Lambda}\right)^{*}, S_{b} \subset S_{b}^{*}$, and $S_{b}^{\Lambda} \subset\left(S_{b}^{\Lambda}\right)^{*}$. Since $E \cap S_{b}=\{0\}\left(\subset S_{b}^{*}\right)$, the linear hull of the vector spaces $E$ and $S_{b}^{\Lambda}$ in $S_{b}^{*}$ is their direct sum; we denote it by $K_{\mathrm{A}}$. Similarly, for the space $S_{b}$, we set $K=E \oplus S_{b}\left(\subset S_{b}^{*}\right)$.

If $z=z_{1}+z_{2} \in K\left(\subset S_{b}^{*}\right), z_{1} \in K, z_{2} \in S_{b}$, and $a \in S_{b}$, then $\langle z, a\rangle=a\left(z_{1}\right)+z_{2}(a)$.

The situation considered above can be described by the relations

$$
E^{\prime} \supset H \supset E, \quad E^{\prime} \supset S_{b}, \quad E \oplus S_{b}=K \subset \dot{S}_{b}^{*} \supset S_{b}^{\prime} \supset S_{b} ;
$$

similar relations hold for $S_{b}^{\Lambda}$.

Definition 5. The Lévy-Gauss measure corresponding to a basis $b$ and a countable dense subset $\Lambda \subset \mathbb{R}^{1}$ is the Gaussian $S_{b}^{\Lambda}$-cylindrical measure (defined on an arbitrary space dual to $S_{b}^{\Lambda}$, e.g., on $E$, or on $S_{b}^{\Lambda}$, or on $K$ ) whose correlation functional coincides with the inner product $(\cdots)_{\mathrm{PL}}$. The $S_{b}$-cylindrical Lévy-Gauss measure $\nu_{\mathrm{PL}}^{b}$ is defined similarly (we sometimes denote both measures by $\nu_{\mathrm{PL}}$ ).

The origin of the Lévy-Gauss measure is described below.

Theorem 4. Suppose that $\Lambda$ is a countable dense subset of $\mathbb{R}^{1}$ and

$$
a_{\lambda}=\sum_{n=1}^{\infty} \cos \lambda n \cdot e_{n}
$$

for each $\lambda \in \Lambda$. Then the vector space $\bar{S}_{b}^{\Lambda}$ of all sequences

$$
\left(\alpha_{j} a_{\lambda_{j}}: j \in \mathbb{N}, \lambda_{j} \in \Lambda, \sum_{j=1}^{\infty} \frac{\alpha_{j}^{2}}{j^{2}}<\infty\right)
$$

( $S_{b}^{\Lambda}$ is canonically embedded in this space) is a Hilbert support of the measure $\nu_{\mathrm{PL}}^{b \Lambda}$; the Hilbert norm on $\bar{S}_{b}^{\Lambda}$ is defined by

$$
\left\|\left(\alpha_{j} a_{\lambda_{j}}\right)\right\|^{2}=\sum_{j=1}^{\infty} \frac{\alpha_{j}^{2}}{j^{2}}
$$

Theorem 4 follows from Theorem 3.

Remark 6. The analog of Theorem 4 for the measure $\nu_{\mathrm{PL}}^{b}$ is invalid: by Proposition 1, this measure has no Hilbert support.

On the other hand, the following proposition is valid.

Proposition 2. Suppose that $\lambda \in \mathbb{R}^{1}$ and $\bar{S}_{b}$ is the vector space of all sequences

$$
\left(\alpha_{j} a_{\lambda_{j}}: j \in \mathbb{N}, \alpha_{j} \in \mathbb{R}^{1}, \lambda_{j} \in \mathbb{R}^{1}\right),
$$

be represented as a vector subspace of $S_{b}^{*}$ by virtue of the relation

$$
\left(\sum_{j=1}^{\infty} \alpha_{j} a_{\lambda_{j}}\right)\left(\sum_{j=1}^{\infty} d_{j} a_{\lambda_{j}}\right)=\sum_{j=1}^{n} \alpha_{j} d_{j}\left(a_{\lambda_{j}}, a_{\lambda_{j}}\right) \mathrm{PL} .
$$

Then $\bar{S}_{b}$ is a support of the measure $\nu_{\mathrm{PL}}^{b}$.

Proposition 2 follows from Theorem 2.

If $E$ is represented as a vector space of some functions (on a domain in Euclidean space), then $\bar{S}_{b}$ and $\bar{S}_{b}^{\Lambda}$ can be represented as vector spaces of (some) distributions. 
Example 3. Suppose that $E=W_{2}^{1}[0, \pi], H=L_{2}(0, \pi)$, and, for each $k \in \mathbb{N}, e_{k} \in E$ and $e_{k}(t)=$ $c_{k} \sin \pi k t$. Consider the vector subspace

$$
E_{1}=\left\{\sum \beta_{j} a_{\lambda_{j}}, j \in \mathbb{N}, \lambda_{j} \in \Lambda, \sum j^{2} \beta_{j}^{2}<\infty\right\}
$$

of $H$ (we use the notation and assumptions of Theorem 4).

Let $\|\cdot\|_{1}$ be the Hilbert norm on $E_{1}$ defined by $\|\cdot\|_{1}=\left\|\sum_{j=1}^{\infty} j^{2} \beta_{j}^{2}\right\|$. The inequality $\sum j^{2} \beta_{j}^{2}<\infty$ implies the convergence of the series $\sum\left|\beta_{j}\right|$ and therefore, the inclusion $E_{1} \subset E$; in addition, $E_{1}$ is contained in the completion $S$ of the space $\left(S_{b}^{\Lambda},\|\cdot\|_{\mathrm{PL}}\right)$, and the canonical embedding $E_{1} \rightarrow S$ is Hilbert-Schmidt; thus $S$ is embedded (by a Hilbert-Schmidt map) in a Hilbert space $E_{1}^{\prime}$, which is a support of the measure $\nu_{\mathrm{PL}}$ (actually, the space $E_{1}^{\prime}$ is isomorphic to the space $\bar{S}_{b}^{\Lambda}$ ). Thus the support of the measure $\nu_{\mathrm{PL}}$ can be represented as the dual space of a subspace $E_{1}$ of the space $E^{\prime}$; this space consists of some distributions on $[0, \pi]$, so the elements of the subspace can also be called distributions.

\section{§4. Lévy Laplacians, Lévy traces, and related objects}

This section describes the origin of the Lévy-Gauss measure.

Let $\Phi$ be the vector space of twice Fréchet differentiable functions on a Hilbert space. Then the secondorder differential operator $\Delta^{\prime}$ on $\Phi$ specified by a linear (continuous) functional $l$ on the space $L(H)$ of linear continuous operators in $H$ is defined by $\left(\Delta^{\prime} f\right)(x)=l\left(f^{\prime \prime}(x)\right)$. If $l(A)=\operatorname{tr}(B A)$, where $B$ is a trace class operator in $H$, then $\Delta^{\prime}$ is called the Volterra-Gross Laplacian; if the restriction of $l$ to the set of operators of the form $\alpha \cdot \operatorname{Id}+D$ (where Id is the identity map, $\alpha \in \mathbb{R}^{1}$, and $D$ is a compact map) is defined by $l(\alpha \cdot \operatorname{Id}+D)=\alpha$, then $\Delta^{l}$ is called the Lévy Laplacian.

We can similarly specify (second-order) differential operators on spaces of functions defined on dense subsets of a Hilbert space.

Let $E, H$, and $b$ be the same as in the preceding section, and let $\Phi$ be the vector space of functions on $E$ twice Gateaux differentiable at each point. Consider the linear functional $l$ defined on some subspace $L_{0}$ of the space of all linear maps from $E$ into $E^{\prime}$ by the formula

$$
l(A)=\lim _{n \rightarrow \infty} \frac{1}{n} \sum_{j=1}^{n}\left(A e_{j}, e_{j}\right)
$$

it is assumed that $L_{0}$ consists of all operators for which the limit on the right-hand side in this equation exists. The functional on $L_{0}$ defined in this way is called the Lévy trace and denoted by trpL (of course, the Lévy trace and its domain depend on the choice of the basis $b$ ). In what follows, we denote the Lévy Laplacian by $\Delta_{\mathrm{PL}} ;$ thus $\Delta_{\mathrm{PL}} f(x)=\operatorname{tr}_{\mathrm{PL}} f^{\prime \prime}(x)$ for $f \in \Phi$. To solve the equation

$$
\frac{\partial f}{\partial t}=\frac{1}{2} \Delta_{\mathrm{PL}} f
$$

with respect to the function $f:[0, \infty) \times E \rightarrow \mathbb{R}^{1}$ by the method of Fourier transform (for the first time, this was done in [3]), it is useful to apply a fundamental solution of this problem, i.e., (some) solution of the equation

$$
\frac{\partial \nu(t)}{\partial t}=\frac{1}{2} \Delta \mathrm{PL} \nu(t)
$$

for a function $\nu$ of real argument ranging in a space of (some) measures on $E$. It can be shown that these measures are $G$-cylindrical for a suitable space of linear functionals on $E$, and the operator $\Delta_{\mathrm{PL}}$ is defined as the pseudodifferential operator corresponding to the Lévy inner product with the sign reversed.

Thus the Fourier transform reduces (1) to the equation

$$
\frac{\partial \widetilde{\nu}(t)}{\partial t}=-\frac{1}{2}(x, x)_{\mathrm{PL}} \widetilde{\nu}(t)
$$


$(\tilde{\nu}=F \nu)$ for a function ranging in the space of functions on $S_{b}^{\Lambda}$. The solution of the Cauchy problem for this equation with initial data $(0,1)$ is the function specified by $\widetilde{\nu}(t)(x)=e^{-\frac{t}{2}(x, x)_{\mathbf{P L}}} ;$ but $\widetilde{\nu}(t)(\cdot)$ is just the Fourier transform of the Lévy-Gauss measure.

Let us make several additional remarks about the relation between the usual (Volterra-Gross) Laplacian and the Lévy Laplacian and between integration with respect to the Lévy-Gauss measure treated as an $S_{b}^{\Lambda}$. cylindrical measure on $E$ and integration with respect to its countably additive version, which is a measure on $\bar{S}_{b}^{\Lambda}$.

Let $\psi$ be a function on $S_{b}^{\Lambda}$ that is the Fourier transform of some $S_{b}^{\Lambda}$-cylindrical measure $\nu$ on $E$ (we are interested in the case where $\nu$ is the Lévy-Gauss measure). As was mentioned above, on each space dual to $S_{b}^{\Lambda}$ we can then define exactly one $S_{b}^{\Lambda}$-cylindrical measure with the same Fourier transform; it is natural to apply the term Lévy-Gauss measure to these measures too. On the other hand, if $\sigma_{E}$ is the $\sigma$-algebra of subsets of $S_{b}^{\wedge}$ that is generated by $E$-cylindrical sets, then, as can readily be verified, $\sigma_{E}$ coincides with the $\sigma$-algebra generated by $\left(S_{b}^{\Lambda}\right)^{\prime}$-cylindrical sets. Thus, the Fourier transform of a countably additive measure on $\sigma_{E}$ can be defined as a function on either $E$ or $\left(S_{b}^{\Lambda}\right)^{\prime}$; the uniqueness of a measure with a given Fourier transform implies that the Fourier transform of the countably additive measure defined on the algebra of $E$-cylindrical sets (the transform is a function on $E$ ) uniquely determines a function on $\left(S_{b}^{\Lambda}\right)^{\prime}$ whose (inverse) Fourier transform coincides with the restriction to $\mathfrak{A}\left(S_{b}^{\Lambda},\left(S_{b}^{\Lambda}\right)^{\prime}\right)$ of this measure extended to $\sigma_{E}$.

Suppose that $\nu$ is a countably additive measure on $\sigma_{E}, F_{E} \nu$ is the Fourier transform of its restriction to $\mathfrak{A}\left(S_{b}^{\Lambda}, E\right)$ (so $F_{E} \nu$ is a function on $E$ ), and $F_{S} \nu$ is the Fourier transform of its restriction to $\mathfrak{A}\left(S_{b}^{\Lambda}, S_{b}^{\Lambda}\right)$ (so $F_{S \nu}$ is a function on $S_{b}^{\Lambda}$ ); suppose also that

$$
\int\|x\|_{\mathrm{PL}}^{2} \nu(d x)<\infty
$$

and $\Delta$ is the Volterra-Gross operator on the space of functions on $S_{b}^{\Lambda}$. Then the functions $\Delta\left(F_{S} \nu\right)$ and $\Delta_{\mathrm{PL}}\left(F_{E} \nu\right)$ are everywhere defined and can be expressed in terms of each other.

Now note that if a function $f$ defined on a vector space $G$ dual to (some) vector space $Z$ is a limit function for a uniformly convergent sequence of $Z$-cylindrical functions, then on any other vector space $G_{1}$ dual to $Z$ there exists a function that is the uniform limit of "the same" sequence of $Z$-cylindrical functions. (A function $f$ on a space $G$ dual to $Z$ is called $Z$-cylindrical if there exist $n \in \mathbb{N}, z_{1}, \ldots, z_{n} \in Z$, and $\varphi: \mathbb{R}^{n} \rightarrow \mathbb{R}^{1}$ such that $f(g)=\varphi\left(\left\langle g, z_{1}\right\rangle, \ldots,\left\langle g, z_{n}\right\rangle\right)$.

Thus, to integrate a function that is defined on $E$ and is the uniform limit of a sequence of $S_{b}^{\Lambda}$. cylindrical functions with respect to the Lévy-Gauss measure, we can find a function that is defined on the support of the Lévy-Gauss measure and is the limit function for "the same" sequence of $S_{b}^{\Lambda}$-cylindrical functions and declare the integral of this function with respect to the (countably additive) Lévy-Gauss measure to be the integral of the original function with respect to the (non-countably additive) Lévy-Gauss measure. It can be verified that this definition is independent of the choice of the convergent sequence of cylindrical functions. Of course, instead we could integrate every cylindrical function from the initial sequence with respect to the noncountably additive Lévy-Gauss measure, prove that the sequence of the obtained integrals converge, and declare its limit, which does not depend on the sequence of cylindrical functions, to be the integral of the limit function with respect to the noncountably additive measure. Nevertheless, inclusion of the Lévy-Gauss measure in the standard Lebesgue theory is useful.

Note that the integral with respect to the Lévy-Gauss measure of a function that is the Fourier transform of a countably additive measure on $\left(S_{b}^{\Lambda}, \sigma_{E}\right)$ can be determined via the Parseval identity; this identity is proved with the use of the notion of support of a Lévy-Gauss measure.

To conclude, we give several formulas related to the Lévy Laplacian. We use the notation of $\S 3$.

(1) For a linear map $A: E \rightarrow E^{\prime}$, the Lévy-Fredholm determinant is $\operatorname{det}_{\mathrm{PL}} A=e^{\operatorname{tr}_{\mathrm{PL}} \ln A}$. It can be shown that if this determinant exists, then

$$
\operatorname{det}_{\mathrm{PL}} A=\lim _{n \rightarrow \infty}\left(\operatorname{det}\left|A e_{j}\left(e_{k}\right)\right|\right)^{1 / n}, \quad j, k=1,2, \ldots, n
$$


(2) If $A$ is a trace class operator in $S_{b}^{\Lambda}$ and $A^{*}$ is the map from $E$ into $S_{b}^{\Lambda} \subset E^{\prime}$ specified by $(A x)(z)=\left(x, A^{*} z\right)_{\mathrm{PL}}$, then $\operatorname{tr}_{\mathrm{PL}} A^{*}=\operatorname{tr} A$ (this formula was implicitly used above) and $\operatorname{det} \mathrm{PL} A^{*}=\operatorname{det} A$ (the right-hand side is the usual Fredholm determinant).

(3) The Lévy-Fredholm-Carleman determinant can be defined similarly.

(4) If $a, b \in S_{b}^{\Lambda} \subset E^{\prime}$, then

$$
\int_{E} a(x) b(x) \nu_{\mathrm{PL}}(d x)=(a, b) \mathrm{PL} .
$$

L. Accardi acknowledges partial support of the Russian Foundation for Basic Research under grant No. 96-01-00030.

\section{References}

1. I. Ya. Aref'eva and I. V. Volovich, "Functional laws of preservation of higher orders in gauge theories," in: Distributions and Their Applications to Mathematical Physics [in Russian], Steklov Mathematics Institute, Moscow (1981), pp. 43-50.

2. L. Accardi, P. Gibilisco, and I. V. Volovich, "Yang-Mills gauge fields as harmonic functions for the Lévy Laplacian," Russian J. Math. Phys., 2, No. 2, 235-250 (1994).

3. L. Accardi, P. Rozelli, and O. G. Smolyanov, "Brownian motion generated by the Lévy Laplacian," Mat. Zametki [Math. Notes], 54, No. 5, 144-149 (1993).

4. L. Accardi and O. G. Smolyanov, "The Gaussian process generated by the Lévy Laplacian and the corresponding Feynman-Kac formula," Dokl. Ross. Akad. Nauk [Russian Acad. Sci. Dokl. Math.], 342, No. 4, 442-446 (1995).

5. L. Accardi and O. G. Smolyanov, "Transformations of Gaussian measures generated by the Lévy Laplacian and generalized traces," Dokl. Ross. Akad. Nauk [Russian Acad. Sci. Dokl. Math.], 342, No. 5, 234-237 (1995).

6. L. Accardi and O. G. Smolyanov, "On Laplacians and traces," Confer. Sem. Mat. Univ. Bari, 250, 1-25 (1993).

7. O. G. Smolyanov and S. V. Fomin, "Measures of topological linear spaces," Uspekhi Mat. Nauk [Russian Math. Surveys], 31, No. 4, 1-76 (1976).

8. Yu. L. Daletskii and S. V. Fomin, Measures and Differential Equations on Infinite-Dimensional Spaces [in Russian], Nauka, Moscow (1983).

M. V. Lomonosov Moscow State University

Translated by O. V. Sipacheva 